Estudios de la Paz y el Conflicto

Revista Latinoamericana

IUDPAS-UNAH

Número Especial, pp. 120-126

ISSN-e: 2707-8922 / ISSN: 2707-8914

DOI: $10.5377 /$ rlpc.v0i0.9508

Anual 2019

\title{
EXPERIENCIAS DEL SEGUNDO CAMPAMENTO DE JÓVENES CONSTRUCTORES DE PAZ CLAIP
}

\author{
EXPERIENCES OF THE SECOND YOUTH PEACEBUILDING CAMP
}

\author{
Alfonso Hernández Gómez \\ Global Unity Sumak Kausay \\ ahimsamusik@gmail.com
}

Diana Marcela Agudelo Ortiz

Universidad Externado de Colombia

diana.agudelo@uexternado.edu.co

\section{INTRODUCCIÓN}

El Consejo Latinoamericano de Investigación para la Paz -CLAIP- convocó y acogió entre los días 15, 16 y 17 de septiembre de 2019, en la provincia de San Roque, estado de Sao Paulo, Brasil, el II Encuentro de la Red de Jóvenes Constructores de Paz de América Latina, dando continuidad al espacio iniciado en el encuentro anterior de CLAIP México 2017, en el que se dio cabida a esta iniciativa en virtud de la urgencia de la apertura de los diálogos suscitados por el espacio de encuentro académico hacia el espacio vital de nuestras comunidades, sus desafíos y exigencias. Se propuso, entonces, un espacio para la configuración y consolidación de una red de trabajo latinoamericana para el intercambio activo y dialógico entre la juventud, que ayude a ampliar las perspectivas de futuro en distintas áreas de trabajo a partir de la diversidad de experiencias, aprendizajes y metodologías desarrolladas por los participantes, todo esto encaminado hacia la construcción de paz transformadora en el continente. Este es un breve relato de lo que vivimos en el Campamento.

\section{EL ENCUENTRO Y SU DESARROLLO}

Este sueño se sembró durante la sesión del CLAIP desarrollada en el marco de la Conferencia General de IPRA en Ahmedabad - India en diciembre de 2018. Desde allí comenzamos a imaginar un espacio distante del ruido de la cotidianidad citadina para lo que sería nuestra premisa de allí en adelante: conectar(nos), articular(nos), tejer(nos). Una vez escogido Brasil como sede del encuentro de CLAIP, pensamos en la playa, en el bosque, en un parque natural... hasta que la palabra del Primer Encuentro de los jóvenes del CLAIP en la Ciudad 
de México en 2017 se materializó en un espacio inmejorable en Novo Horizonte, muy cerca de São Roque en el estado de São Paulo. Nuestro equipo de trabajo entonces se dispuso a sumar en este telar desde sus distintas latitudes, ajustando horarios entre Buenos Aires, Sao Paulo, Bogotá, Tegucigalpa y Ciudad de México. Y fue así que a través de las palabras, las risas y los deseos de juntanzas, finalmente se cocinó este encuentro.

Así fueron llegando desde Colombia, México y otras ciudades de Brasil 30 jóvenes cargados de ideas y deseos de transformación a la cita del 15 de septiembre en la estación de trenes más grande y concurrida de São Paulo: Barra Funda. Se respiraba una atmósfera de emoción mezclada de desconcierto, de prisas nerviosas, cansancio alegre, y de conversaciones que nos hacían brillar los ojos.

Desde el principio propusimos la importancia de abrirle espacios al sentir, al movimiento corporal y al juego, que son tan necesarios para nuestro horizonte común como el pensar, el diálogo y las discusiones colectivas. La forma en la que se desarrolló el encuentro responde a una práxis sentipensante, basada en una formación que incluye el pensamiento, la palabra, la escucha, el sentir, el cuerpo, lo anímico y lo energético, inspirada en distintas metodologías participativas. El contacto con la naturaleza, con uno mismo y con el otro fueron la base desde la cual se diseñaron las actividades. En palabras de una de las participantes:

"El escenario del encuentro no pudo ser mejor. Fue un lugar lleno de verdes y azules, un lugar que nos acercó a la paz, logramos la conexión con la naturaleza y [...] que nos apartáramos del exterior y a su vez que nos acercáramos más entre nosotros, que privilegiáramos las conversaciones entre nosotros y cada momento fue una rica experiencia de intercambio $y$ crecimiento" (Ana Fernanda Marulanda - Colombia).

Quizás uno de los invitados más entusiastas con el encuentro, fue justamente el Secretario General de IPRA, Matt Meyer, quien reafirmó la presencia de los jóvenes como una de las claves para el futuro de los colectivos y organizaciones como CLAIP, y para la construcción de paz en el mundo en términos amplios. Su presencia en el campamento aportó a las inquietudes, expectativas y preguntas de los más jóvenes, la posibilidad de poner en diálogo su experiencia y perspectiva respecto a los muchos movimientos que estamos viendo en la actualidad en el mundo, y el cómo los movimientos sociales están afrontando retos que ni siquiera habían sido imaginados en el pasado (ver imagen 1).

Desde el inicio del encuentro las inquietudes e intervenciones giraron en torno a la relación entre la necesidad del cambio social y los principios de la noviolencia, la efectividad de la lucha noviolenta en contextos armados caracterizados por el desconocimiento de derechos humanos y/o el narcotráfico, así como el aterrizaje de estas inquietudes en contextos específicos como el post acuerdo de paz en Colombia, y el escenario de las fronteras, migraciones y el caso del muro del presidente Trump. Uno de los elementos que más fuerza fu tomando tuvo que ver con la necesidad sentida de innovar las formas de acción y protesta. "Make revolution as non-violent as possible, and make nonviolence as revolutionary as possible" (Matt Meyer) sería el resumen perfecto para la maravillosa conversación intergeneracional del primer día. 


\section{Imagen 1. Jóvenes participantes del II Campamento de Jóvenes Constructores de Paz CLAIP}

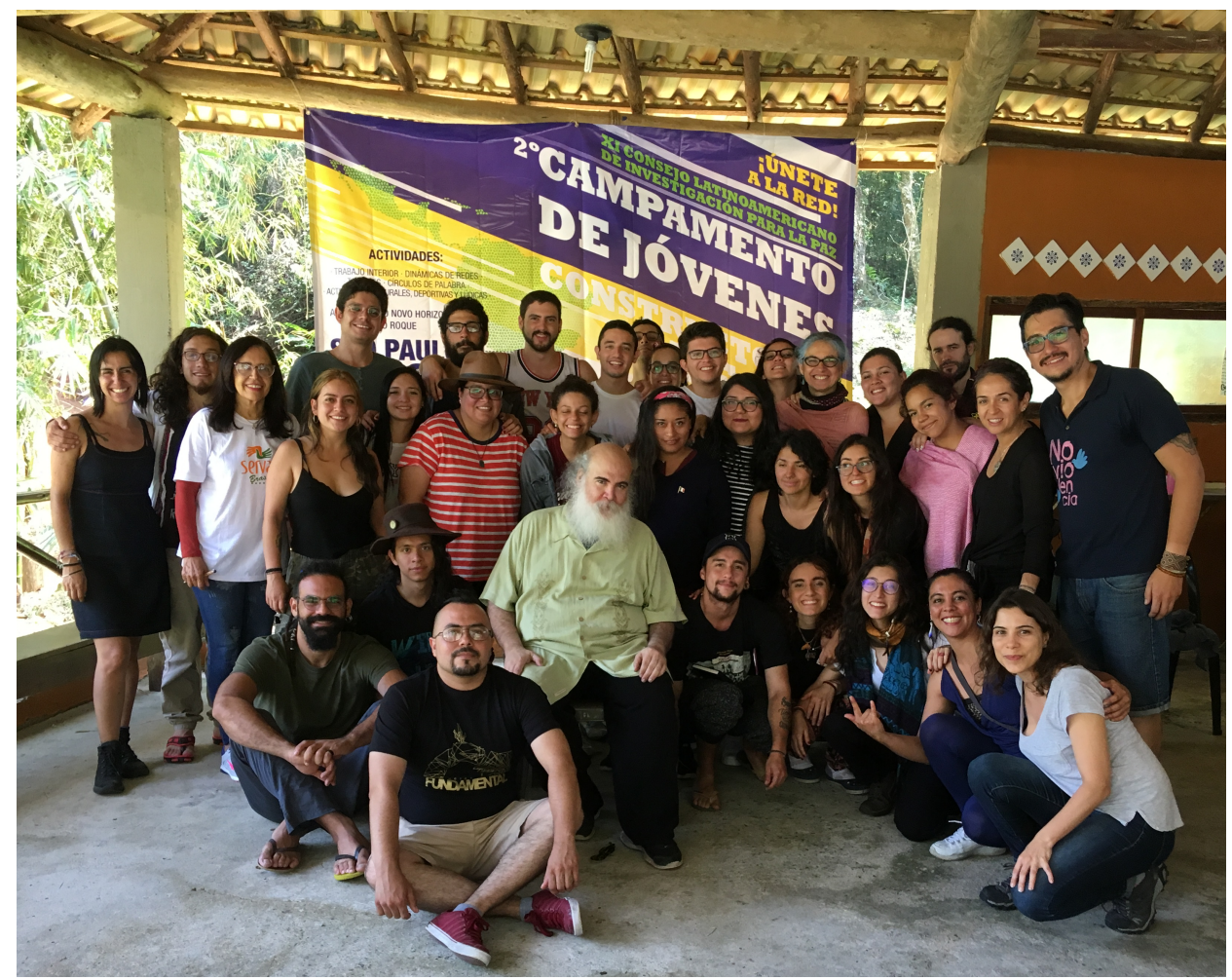

La agenda del campamento continuó hacia el Mapeo de las violencias en América Latina (ver imagen 2). Este ejercicio permitió hacer evidente el contorno de las regiones y países donde se presentan los casos de violencia, pero sobretodo, las interconexiones entre ellos en la dinámica regional de nuestro continente. Resaltaron en particular fenómenos como los flujos de las poblaciones migrantes, los circuitos de tráfico de personas y de drogas y cómo estos afectan a determinadas poblaciones en sus vidas diarias, como en los casos de las zonas de conflicto territorial en México y Colombia atravesando todo Centroamérica. Eso nos permitió ver la importancia de pensar los problemas y las soluciones de forma interconectada, como lo señala otra de las participantes:

"Mapear las violencias de nuestros territorios, tomando en cuenta los países representados, se convirtió en un zoom a nuestra cotidianeidad; a mi realidad. ¿Qué esperar de la niñez y las juventudes si viven violentados constantemente por todo lo que les rodea? ¿Cómo he logrado salir de ese círculo de violencia, siendo que también ha sido mi realidad? ¿En verdad lo he logrado?”

(Daniela Sepúlveda - México).

Otros escenarios problemáticos que sobresalieron en nuestro mapeo están relacionados con la agroindustria y la minería extractiva (legal e ilegal) como materia prima para la cadena de producción económica mundial, así como el despojo de tierras asociado a esa dinámica extractivista; los impactos del cambio climático; las conexiones entre nuestros Estados patriarcales, capitalistas, clasistas-excluyentes-racistas; una violencia cultural extensa y manifiesta en fenómenos como el individualismo y la indiferencia; y en general las múltiples formas de vulneración que recaen sobre la juventud: aumento de violencia/pandillerismo y bandas criminales (matarnos entre nosotros); la identidad "en construcción" de uno frente al Estudios de la Paz y el Conflicto, Revista Latinoamericana, IUDPAS-UNAH, Número Especial 1, 2019, pp. 120-126. DOI: $10.5377 /$ rlpc.v0i0.9508 
otro ("sálvese quien pueda"), microtráfico/consumo de estupefacientes como estrategia de adormecimiento. Todos estos ejes producen múltiples violencias que se interrelacionan y retroalimentan.

\section{Imagen 2. Mapeo de violencias en América Latina}

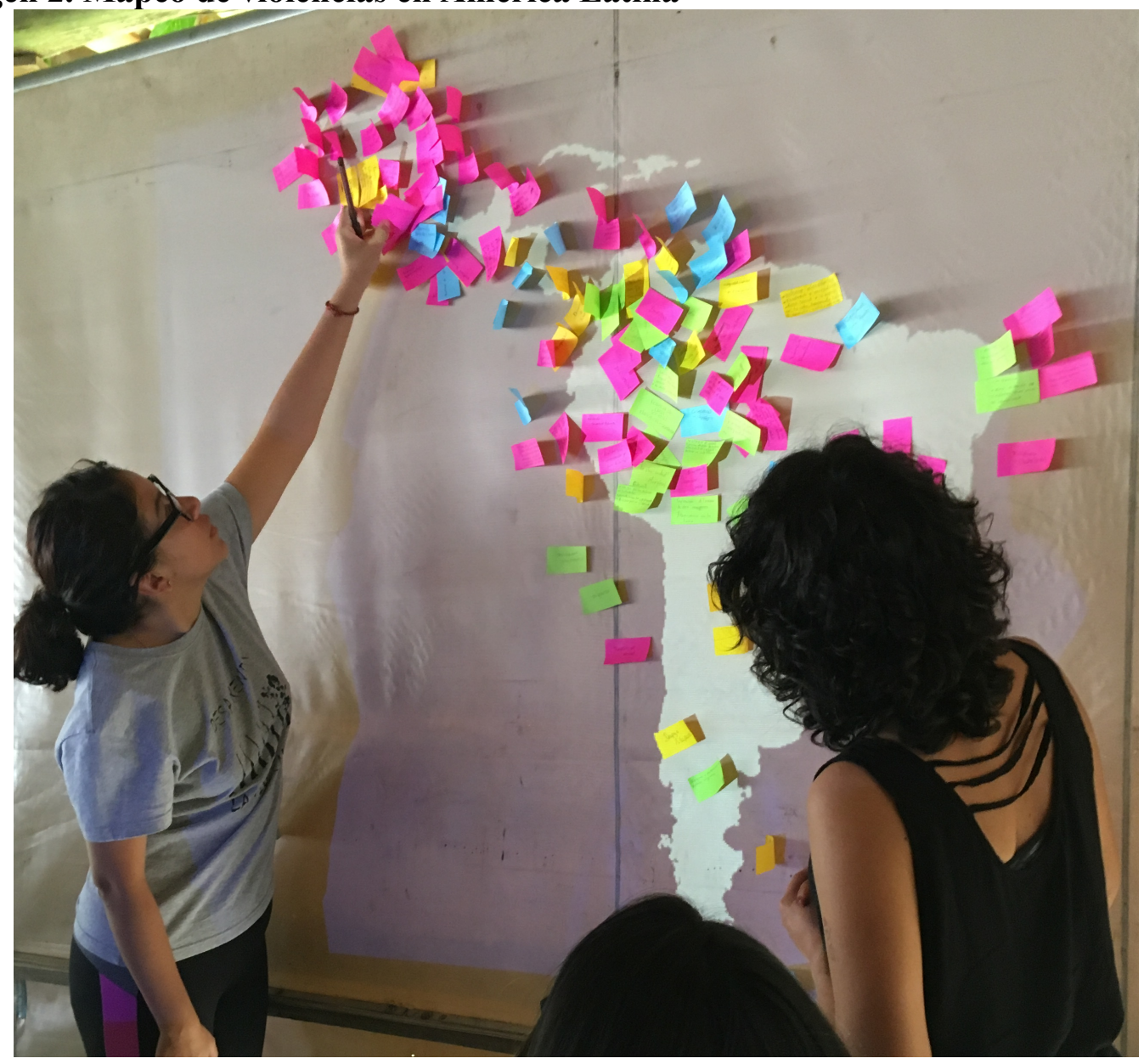

De esta forma llegamos a una de las conclusiones más importantes para el desafío de futuro de la red, que tiene que ver con que la construcción de paz y la noviolencia también deberán articularse de manera conjunta y colectiva, ya que requiere del trabajo conjunto en distintos países ante las interconexiones de estas problemáticas. El camino hacia el Mapeo acerca de las experiencias de construcción de paz en el continente, incluyendo nuestras propias acciones es desde ya una tarea de cara a Honduras 2021.

Uno de los momentos más sentipensantes del espació consistió en la exploración de nuestras propias historias como sujetos latinoamericanos. Un ejercicio de círculo de reflexión (ver imagen 3) abrió la puerta a otro registro de esas mismas violencias, pero ya no desde afuera sino desde nuestros propios entramados vitales. Esta metodología inspirada en las vertientes del pensamiento crítico latinoamericano implicó otra forma de acercarnos a la comprensión de lo que implica la construcción de paz para la historia de nuestros países:

"As atividades e oficinas no geral foram incriveis e pudemos absorver muito conhecimento, mesmo nos momentos mais intimistas e pessoais foi possível aprender sobre a América Latina". (Jeannine Loyola - Brasil).

Estudios de la Paz y el Conflicto, Revista Latinoamericana, IUDPAS-UNAH, Número Especial 1, 2019, pp. 120-126. DOI: $10.5377 /$ rlpc.v0i0.9508 


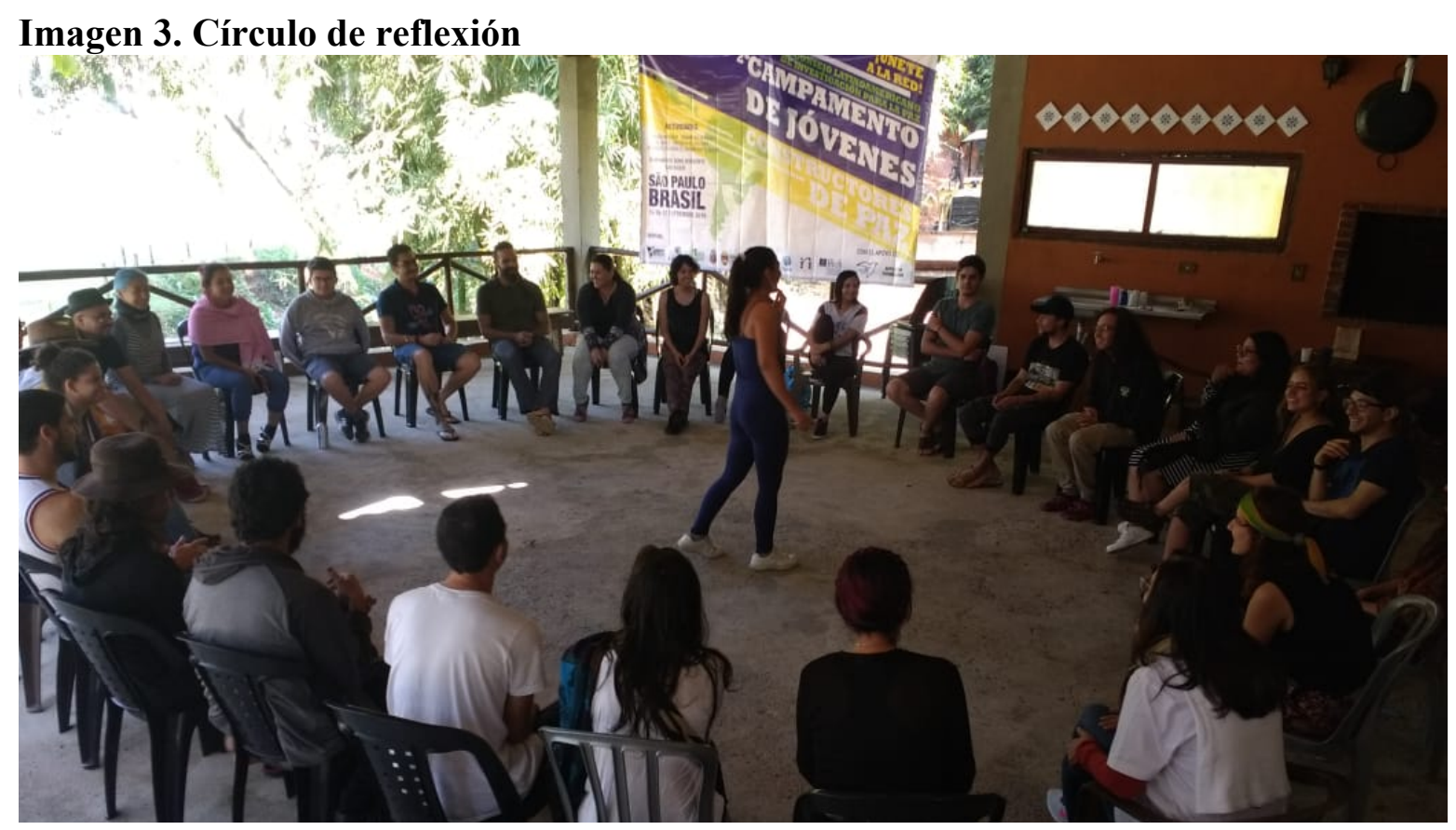

La nostalgia, el dolor y la ternura fueron el hilo de un vínculo que parecía robustecerse con cada historia. Esa experiencia nos permitió conectar, trascender la teoría, la academia y lo utilitario, para llevarnos de lleno al corazón, que es donde la paz surge. Transitamos así, juntos, de nuestras narraciones de dolor hacia la esperanza colectiva de sanar y restaurar. Así lo relata una de las participantes:

"En esa revolución de emociones un abrazo puede cambiar tu estado de ánimo, pero un cúmulo de abrazos de quienes hacen lo posible por cambiar la violenta realidad en la que estamos envueltos y de la que somos parte, no sólo deja una sensación de alivio, sino que crea un lazo de esperanza, un vínculo que va más allá de la intimidad que hace posible crear un nudo de complicidad dificil de desatar" (Daniela Sepúlveda - México).

Dos actividades más abrirían el campo para los encuentros y las posibilidades de acción común: el documental "Brave Now World - Un Viaje Raíz" de Hermanos - Brothers, y los ejercicios corporales del grupo Extémpora Danza, nos dieron elementos hacia una inmersión poética por las venas de nuestro cuerpo y nuestro planeta. Las palabras de María Cano retumbaron en nuestros oídos una y otra vez: "Hijos de virtuosos campesinos y abnegados obreros, ¿cómo pueden disparar al pecho de sus hermanos?" La frase comenzó a recorrer nuestra voz, nuestra piel, los sentidos y la memoria, dejando una marca indeleble, que trascendía e invocaba otros tiempos y otros cuerpos.

Las actividades que siguieron encontraron su propio ritmo, pues tras haber pasado día y medio compartiendo, aún no se trabajaba sobre lo que cada uno hacía. Intencionalmente diseñado para romper con la dinámica de muchos encuentros académicos en los que las personas solo se hacen presente para hablar de la importancia de su trabajo, el prolongar este momento permitía que el deseo de escuchar y conocer al otro se hiciera manifiesto. Ya no se trataba de un monólogo, sino de un diálogo deseado, urgido. 
Respondiendo las cuestiones acerca de 1) Áreas de incidencia y acción; 2) Problemática abordada; 3) Acción desarrollada y; 4) Agentes involucrados, fuimos avanzando hacia el descubrimiento de las posibles convergencias y las lecciones aprendidas. La riqueza de las experiencias aportadas por los jóvenes nos dejó más que entusiasmados: el arte, la cultura, la academia y el andamiaje institucional cubrieron el espectro de posibilidades allí reunidas.

Los colectivos y proyectos se cruzaban en la búsqueda de incidencia sobre dinámicas como la violencia de género, el arte como herramienta de transformación social para la juventud, así como distintas perspectivas acerca del trabajo con la memoria hacia la acción colectiva. El uso de las redes sociales y las nuevas tecnologías de la comunicación serían las herramientas principales de cada uno de los proyectos que se propusieron. En palabras de otro de los participantes:

"Las formas comunes desde las cuales nos pudimos encontrar hacen referencia a la música, el arte y la expresión, encarnadas en unos protagonistas concretos que refieren a la juventud como los encargados de llevar la batuta en esta orquesta de paz. Paz y juventud son dos palabras cuyo significado debe estar contextualizado en materialidades que permiten poner estas dos palabras en una misma oración, en un mismo espacio tiempo y crear realidades en donde los jóvenes somos parte de la paz más allá del discurso y el cliché” (Mateo Rico - Colombia).

Finalmente, tras un acercamiento al contexto de algunas de las movilizaciones estudiantiles y de ocupación en Brasil, planteamos la construcción de una visión común y de unos principios con los cuales identificaremos el trabajo de esta Red de Jóvenes CLAIP. Mediante un ejercicio de prospectiva y la elaboración de un Mandala, fuimos contestando preguntas como: ¿cómo vemos el futuro de nuestros países y de toda la región? ¿qué papel jugamos en ese futuro? ¿cómo eso conecta con nuestras propias vidas y expectativas? ¿cómo encajar el cambio social con los valores de vida que comparto y con la no-violencia? ¿qué hacer para avanzar hacia una paz transformadora y participativa en medio de la incertidumbre propia de nuestros contextos? Así, avanzamos sobre nuestra visión:

"Desde la unión, la solidaridad, el amor, la cooperación y la esperanza, construimos caminos hacia un mundo sin fronteras, trascendiendo en la palabra y la acción, reconociendo nuestras diferencias y potencializando nuestras fortalezas". Red de Jóvenes CLAIP, 2019.

En resumen, el campamento fue una experiencia extraordinaria para quienes participamos en él. Una visión colectiva y solidaria hacia la construcción de paz con perspectiva transformadora y participativa no puede prescindir de diálogos intergeneracionales, ni de la articulación de las visiones que se construyen dentro, fuera y en contra de las instituciones, que de acuerdo al contexto, pueden ser útiles tanto a las dinámicas de violencia como a las dinámicas de resistencia y transformación de nuestros países. Una lógica sentipensante, aterrizada, historizada y compasiva es el reclamo de todas las voces allí reunidas.

No solo conocimos la forma en la que los jóvenes plantean el cambio social, nos aproximamos a caminos de noviolencia, en los que queda claro que las armas no han sido ni son el camino, que la palabra es más poderosa, que el arte derrumba fronteras y que el amor es la fuerza más poderosa para transformar el mundo. El uso de las nuevas tecnologías aparece como posibilidad para cualquier esfuerzo de construir red, reconociendo que los ámbitos online y off line se alimentan mutuamente, y que el lenguaje de las nuevas generaciones está muy marcado por dicha realidad virtual. Por ello, debemos usarlas a nuestro favor, aprovechar esas tecnologías para poder así potencializar nuestros esfuerzos. 
Los jóvenes están interesados en encontrarse, en tener intercambios culturales y aportar a la realización de proyectos comunitarios. Hay, por una parte, una comprensión contextualizada de las opciones para la construcción de paz, pero también, una aproximación desde la lógica regional y global acerca de las múltiples articulaciones de América Latina con el resto del mundo. La idea de unidad, integración y conexión está muy presente, por lo que es importante abrir y facilitar los espacios para que esto pueda seguir creciendo, ya que a partir de los encuentros, podemos fomentar el liderazgo de los jóvenes, facilitar las sinergias, crear iniciativas conjuntas y generar conocimiento desde esta visión conjunta.

Durante los días de la XI Conferencia General del CLAIP, los jóvenes del campamento aportaron también sus investigaciones, la mayoría de las cuales emergen de sus propias experiencias de trabajo comunitario, de acciones de calle, movimientos sociales o procesos educativos alternativos, como se muestra en algunos de los ensayos de este primer número especial de la Revista. Quedan además en nosotros las huellas de su creatividad y generosidad, que, con cantos, danzas y colores, aportaron nuevas reflexiones para la construcción de paz.

\section{REFERENCIA DE LOS AUTORES}

Alfonso Hernández Gómez, Licenciado en Filosofía por la Universidad Nacional Autónoma de México (UNAM) y Maestro en Ciencias Antropológicas por la Universidad Autónoma de México - Iztapalapa (UAM-I). Candidato a PhD en Ciencias Antropológicas UAM-I. Trabaja en temas de violencia urbana y juvenil, antropología visual y cultura de paz desde el arte. ahimsamusik@gmail.com. ORCID: 0000-0002-3523-9324

Diana Marcela Agudelo Ortiz, Psicóloga. Maestra en Estudios de la Cultura con mención en Políticas Culturales. Candidata a $\mathrm{PhD}$ en Conocimiento y Cultura en América Latina. Docente investigadora del programa de Psicología, el Laboratorio Interdisciplinar de Ciencias y Procesos Humanos (LINCIPH), y del grupo de investigación Salud, conocimiento médico y sociedad de la Facultad de Ciencias Sociales y Humanas, Universidad Externado de Colombia.diana.agudelo@uexternado.edu.co. ORCID: 0000-0002-0729-5708

(c) (1) Licencia Creative Commons Reconocimiento 4.0 (CC BY 4.0).

Estudios de la Paz y el Conflicto, Revista Latinoamericana, IUDPAS-UNAH, Número Especial 1, 2019, pp. 120-126. DOI: $10.5377 /$ rlpc.v0i0.9508 